\title{
Mononen-Karnes-Senac syndrome
}

INSERM

\section{Source}

INSERM. (1999). Orphanet: an online rare disease and orphan drug data base. MononenKarnes-Senac syndrome. ORPHA:2565

Mononen-Karnes-Senac syndrome is characterized by skeletal dysplasia associated with finger malformations (brachydactyly with short and abducted thumbs, short index fingers, and markedly short and abducted great toes), variable mild short stature, and mild bowleg with overgrowth of the fibula. It has been described in two males, their mothers, and a maternal aunt. Females are less severely affected than males. X-linked dominant inheritance is suggested. 